\title{
Some Fuzzy-Wavelet-Like Operators and Their Convergence
}

\author{
R. Ezzati, F. Mokhtarnejad, and N. Hassasi \\ Department of Mathematics, Karaj Branch, Islamic Azad University, Karaj, Iran \\ Correspondence should be addressed to R.Ezzati; ezati@kiau.ac.ir
}

Received 25 January 2013; Accepted 20 May 2013

Academic Editor: Tofigh Allahviranloo

Copyright (C) 2013 R. Ezzati et al. This is an open access article distributed under the Creative Commons Attribution License, which permits unrestricted use, distribution, and reproduction in any medium, provided the original work is properly cited.

\begin{abstract}
Firstly, we define some new fuzzy-wavelet-like operators via a real-valued scaling function to approximate the continuous fuzzy functions of one and two variables. Then, by using the modulus of continuity, we prove their pointwise and uniform convergence with rates to the fuzzy unit operator $I$. Using these fuzzy-wavelet-like operators, we present some numerical examples to illustrate the applicability of the proposed method. Also, we give a new method to approximate the integration of continuous fuzzy realnumber-valued function of two variables by using the fuzzy-wavelet-like operator.
\end{abstract}

\section{Introduction}

Approximating functions in a given space is an old problem. For this purpose, many authors have studied approximation of fuzzy functions on a finite set of distinct points; see [1-6]. For instance, the authors of [2] proposed a new method to approximate fuzzy functions by distance method. Indeed, they considered the problem for fuzzy data and fuzzy functions using the defuzzification function introduced by Fortemps and Roubens. Also, they introduced a fuzzy polynomial approximation as $D$-approximation of a fuzzy function on a discrete set of points.

Wavelet theory is a relatively new and an emerging area in mathematical research. Also, wavelets are the suitable and powerful tool for approximating functions based on wavelet basis functions. In [1], the author defined some fuzzywavelet-like operators and presented their pointwise and uniform convergence with rates to the fuzzy unit operator $I$. Recently, the authors of [7] constructed fuzzy Haar wavelet and applied it on solving linear fuzzy Fredholm integral equation of second kind.

Here, we propose some new fuzzy-wavelet-like operators via a real-valued scaling function to approximate the continuous fuzzy functions of one and two variables. Also, we prove their pointwise and uniform convergence with rates to the fuzzy unit operator $I$ by using modulus of continuity. It is noticed that we do not suppose any kind of orthogonality condition on the scaling function, and the operators act on fuzzy-valued continuous functions over $\mathbb{R}$ and $\mathbb{R}^{2}$.
The rest of the paper is organized as follows. In Section 2, we review some elementary concepts of the fuzzy set theory and modulus of continuity. In Section 3, we prepare some theorems for pointwise and uniform convergence of defined fuzzy-wavelet-like operators with rates to the fuzzy unit operator I. In Section 4, we give two numerical examples for applicability of the proposed methods. In Section 5, we present an application to approximate the integration of continuous fuzzy real-number-valued function of two variables by using the fuzzy-wavelet-like operator. Finally, this paper is concluded in Section 6.

\section{Preliminaries}

Definition 1 (see [8]). A fuzzy number is a function $u: \mathbb{R} \rightarrow$ $[0,1]$ with the following properties:

(1) $u$ is normal; that is, there exists $x_{0} \in \mathbb{R}$ such that $u\left(x_{0}\right)=1$,

(2) $u$ is fuzzy convex set

$$
\text { (i.e., } \begin{aligned}
& u(\lambda x+(1-\lambda) y) \\
\geq & \min \{u(x), u(y)\} \forall x, y \in \mathbb{R}, \lambda \in[0,1]),
\end{aligned}
$$

(3) $u$ is upper semicontinuous on $\mathbb{R}$,

(4) the $\overline{\{x \in \mathbb{R}: u(x)>0\}}$ is compact set.

The set of all fuzzy numbers is denoted by $\mathbb{R}_{F}$. 
Definition 2 (see [9]). Suppose that $u \in \mathbb{R}_{F}$. The $r$-level set of $u$ is denoted by $[u]^{r}=\left[u_{-}^{(r)}, u_{+}^{(r)}\right]$ and defined by $[u]^{r}=$ $\{x \in \mathbb{R} ; u(x) \geq r\}$, where $0<r \leq 1$. Also, $[u]^{0}$ is called the support of $u$, denoted by $\operatorname{supp} u$, and it is given as $[u]^{0}=$ $\overline{\{x \in \mathbb{R} ; u(x)>0\}}$. It follows that the level sets of $u$ are closed and bounded intervals in $\mathbb{R}$.

It is well known that the addition and multiplication operations of real numbers can be extended to $\mathbb{R}_{F}$. In other words, for $u, v \in \mathbb{R}_{F}$, and $\lambda \in \mathbb{R}$, one defines uniquely the sum $u \oplus v$ and the product $\lambda \odot u$ by

$$
[u \oplus v]^{r}=[u]^{r}+[v]^{r}, \quad[\lambda \odot u]^{r}=\lambda[u]^{r}, \quad \forall r \in[0,1],
$$

where $[u]^{r}+[v]^{r}$ means the usual addition of two intervals (as subsets of $\mathbb{R}$ ) and $\lambda[u]^{r}$ means the usual product between a scalar and a subset of $\mathbb{R}$. One uses the same symbol $\sum$ both for the sum of real numbers and for the sum $\oplus$ (when the terms are fuzzy numbers).

Definition 3 (see [9]). An arbitrary fuzzy number is represented, in parametric form, by an ordered pair of functions $(\underline{u}(r), \bar{u}(r)), 0 \leq r \leq 1$, which satisfy the following requirements:

(1) $\underline{u}(r)$ is a bounded left continuous nondecreasing function over $[0,1]$,

(2) $\bar{u}(r)$ is a bounded left continuous nonincreasing function over $[0,1]$,

(3) $\underline{u}(r) \leq \bar{u}(r), 0 \leq r \leq 1$.

The addition and scaler multiplication of fuzzy numbers in $\mathbb{R}_{F}$ are defined as follows:

(1) $u \oplus v=(\underline{u}(r)+\underline{v}(r), \bar{u}(r)+\bar{v}(r))$,

(2)

$$
(\lambda \odot u)= \begin{cases}(\lambda \underline{u}(r), \lambda \bar{u}(r)) & \lambda \geq 0, \\ (\lambda \bar{u}(r), \lambda \underline{u}(r)) & \lambda<0 .\end{cases}
$$

Definition 4 (see [10]). For arbitrary fuzzy numbers $u$, $v$, the quantity

$$
D(u, v)=\sup _{r \in[0,1]} \max \left\{\left|u_{-}^{(r)}-v_{-}^{(r)}\right|,\left|u_{+}^{(r)}-v_{+}^{(r)}\right|\right\}
$$

is the distance between $u$ and $v$. It is proved that $\left(\mathbb{R}_{F}, D\right)$ is a complete metric space with the properties $[11,12]$

(1) $D(u \oplus w, v \oplus w)=D(u, v)$ for all $u, v, w \in \mathbb{R}_{F}$,

(2) $D(k \odot u, k \odot v)=|k| D(u, v)$ for all $u, v \in \mathbb{R}_{F}$ for all $k \in \mathbb{R}$,

(3) $D(u \oplus v, w \oplus e) \leq D(u, w)+D(v, e)$ for all $u, v, w, e \in$ $\mathbb{R}_{F}$.

Definition 5 (see [10]). Let $f, g:[a, b] \rightarrow \mathbb{R}_{F}$ be fuzzy real number valued functions. The uniform distance between $f$, $g$ is defined by

$$
D^{*}(f, g)=\sup \{D(f(x), g(x)) \mid x \in[a, b]\} .
$$

Definition 6 (see [10]). Let $f:[a, b] \rightarrow \mathbb{R}_{F} \cdot f$ is fuzzyRiemann integrable to $J \in \mathbb{R}_{F}$ if for any $\varepsilon>0$, there exists $\delta>0$ such that for any division $P=\{[u, v] ; \xi\}$ of $[a, b]$ with the norms $\Delta(p)<\delta$, one has

$$
D\left(\sum_{P}^{*}(v-u) \odot f(\xi), I\right)<\varepsilon,
$$

where $\sum^{*}$ denotes the fuzzy summation. In this case it is denoted by $I=(F R) \int_{a}^{b} f(x) d x$.

Theorem 7 (see [13]). If $f, g:[a, b] \subseteq \mathbb{R} \rightarrow \mathbb{R}_{F}$ are fuzzy continuous functions, then the function $F:[a, b] \rightarrow \mathbb{R}_{+}$ defined by $F(x)=D(f(x), g(x))$ is continuous on $[a, b]$, and

$$
\begin{gathered}
D\left((F R) \int_{a}^{b} f(x) d x,(F R) \int_{a}^{b} g(x) d x\right) \\
\leq \int_{a}^{b} D(f(x), g(x)) d x .
\end{gathered}
$$

Definition 8 (see [14]). A fuzzy real number valued function $f: \mathbb{R} \rightarrow \mathbb{R}_{F}$ is said to be continuous in $x_{0} \in \mathbb{R}$, if for each $\varepsilon>0$ there is $\delta>0$ such that $D\left(f(x), f\left(x_{0}\right)\right)<\varepsilon$, whenever $x \in \mathbb{R}$ and $\left|x-x_{0}\right|<\delta$. One says that $f$ is fuzzy continuous on $\mathbb{R}$ if $f$ is continuous at each $x_{0} \in \mathbb{R}$ and denotes the space of all such functions by $C_{F}(\mathbb{R})$.

Definition 9 (see [14]). Let $f: \mathbb{R} \rightarrow \mathbb{R}_{F}$. One calls $f$ a uniformly continuous fuzzy real number valued function, if and only if for any $\varepsilon>0$ there exists $\delta>0$ whenever $|x-y| \leq \delta ; x, y \in \mathbb{R}$, implies that $D(f(x), f(y)) \leq \varepsilon$. One denotes it as $f \in C_{F}^{U}(\mathbb{R})$. Also, one denotes the space of all fuzzy continuous functions $f: \mathbb{R}^{2} \rightarrow \mathbb{R}_{F}$ by $C_{F}\left(\mathbb{R}^{2}\right)$.

Similarly we have the following.

Definition 10. Let $f: \mathbb{R}^{2} \rightarrow \mathbb{R}_{F}$. One calls $f$ a uniformly continuous fuzzy real number valued function, if and only if for any $\varepsilon>0$ there exists $\delta>0$ whenever: $\sqrt{\left(x-x_{0}\right)^{2}+\left(y-y_{0}\right)^{2}} \leq \delta ; x, y, x_{0}, y_{0} \in \mathbb{R}$, implies that

$$
D\left(f(x, y), f\left(x_{0}, y_{0}\right)\right) \leq \varepsilon .
$$

One denotes it as $f \in C_{F}^{U}\left(\mathbb{R}^{2}\right)$.

Definition 11 (see [14]). Let $f: \mathbb{R} \rightarrow \mathbb{R}_{F}$, then $f$ is called a nondecreasing function if and only if whenever $x_{1} \leq x_{2}$, $x_{1}, x_{2} \in \mathbb{R}$, one has that $f\left(x_{1}\right) \leq f\left(x_{2}\right)$; that is, $\left(f\left(x_{1}\right)\right)_{-}^{(r)} \leq$ $\left(f\left(x_{2}\right)\right)_{-}^{(r)}$ and $\left(f\left(x_{1}\right)\right)_{+}^{(r)} \leq\left(f\left(x_{2}\right)\right)_{+}^{(r)}$, for all $r \in[0,1]$.

Definition 12 (see $[10,15]$ ). Let $f: \mathbb{R} \rightarrow \mathbb{R}_{F}$ be a bounded function, then function $\omega_{\mathbb{R}}(f, \cdot): \mathbb{R}_{+} \cup\{0\} \rightarrow \mathbb{R}_{+}$,

$$
\omega_{\mathbb{R}}(f, \delta)=\sup \{D(f(x), f(y))|x, y \in \mathbb{R},| x-y \mid \leq \delta\},
$$

where $\mathbb{R}_{+}$is the set of positive real numbers, is called the modulus of continuity of $f$ on $\mathbb{R}$. 
For $f: \mathbb{R}^{2} \rightarrow \mathbb{R}_{F}$, the modulus of continuity $\omega_{\mathbb{R}^{2}}(f, \delta)$ is defined as follows:

$$
\begin{gathered}
\omega_{\mathbb{R}^{2}}(f, \delta)=\sup \left\{D(f(u, v), f(x, y)) \mid(u, v),(x, y) \in \mathbb{R}^{2},\right. \\
\left.\sqrt{(u-x)^{2}+(v-y)^{2}} \leq \delta\right\},
\end{gathered}
$$

where $f \in C_{F}\left(\mathbb{R}^{2}\right)$ and $\delta>0$. Observe that, for all $f \in$ $C_{F}\left([a, b]^{2}\right)$ and $\lambda, \delta>0$, one has

$$
\omega_{[a, b]^{2}}(f, \lambda \delta) \leq(1+[\lambda]) \omega(f, \delta),
$$

where $[\lambda]$ is defined to be the greatest integer less than or equal to $\lambda$.

Some properties of the modulus of continuity are presented below.

Theorem 13 (see [15]). The following properties hold:

(1) $D(f(x), f(y)) \leq \omega_{[a, b]}(f,|x-y|)$ for any $x, y \in[a, b]$,

(2) $\omega_{[a, b]}(f, \delta)$ is increasing function of $\delta$,

(3) $\omega_{[a, b]}(f, 0)=0$,

(4) $\omega_{[a, b]}\left(f, \delta_{1}+\delta_{2}\right) \leq \omega_{[a, b]}\left(f, \delta_{1}\right)+\omega_{[a, b]}\left(f, \delta_{2}\right)$ for any $\delta_{1}, \delta_{2} \geq 0$, and $f: \mathbb{R} \rightarrow \mathbb{R}_{F}$,

(5) $\omega_{[a, b]}(f, n \delta) \leq n \omega_{[a, b]}(f, \delta)$ for any $\delta \geq 0, n \in N$, and $f: \mathbb{R} \rightarrow \mathbb{R}_{F}$

(6) $\omega_{[a, b]}(f, \lambda \delta) \leq[\lambda] \omega_{[a, b]}(f, \delta) \leq(\lambda+1) \omega_{[a, b]}(f, \delta)$ for any $\delta, \lambda \geq 0$, where $[\cdot]$ is the ceiling of the number, any $f: \mathbb{R} \rightarrow \mathbb{R}_{F}$.

(7) If $[c, d] \subseteq[a, b]$ then $\omega_{[c, d]}(f, \delta) \leq \omega_{[a, b]}(f, \delta)$.

In [1], the following theorem is proved.

Theorem 14. Let $f \in C_{F}(\mathbb{R})$ and the scaling function $\varphi(x) a$ real-valued bounded function with $\operatorname{supp} \varphi(x) \subseteq[-a, a], 0<$ $a<+\infty, \varphi(x) \geq 0$, such that $\sum_{j=-\infty}^{\infty} \varphi(x-j)=1$ on $\mathbb{R}$. For $k \in \mathbb{Z}, x \in \mathbb{R}$, put

$$
\left(B_{k} f\right)(x):=\sum_{j=-\infty}^{\infty} f\left(\frac{j}{2^{k}}\right) \odot \varphi\left(2^{k} x-j\right)
$$

which is a fuzzy-wavelet-like operator. Then

$$
\begin{gathered}
D\left(\left(B_{k} f\right)(x), f(x)\right) \leq \omega_{\mathbb{R}}\left(f, \frac{a}{2^{k}}\right), \\
D^{*}\left(B_{k} f, f\right) \leq \omega_{\mathbb{R}}\left(f, \frac{a}{2^{k}}\right),
\end{gathered}
$$

for all $x \in \mathbb{R}$ and $k \in \mathbb{Z}$. If $f \in C_{F}^{U}(\mathbb{R})$, then as $k \rightarrow+\infty$ one gets $\omega_{\mathbb{R}}\left(f, a / 2^{k}\right) \rightarrow 0$ and $\lim _{k \rightarrow+\infty} B_{k} f=f$, pointwise and uniformly with rates.

\section{Main Results}

Theorem 15. All assumption here are as in Theorem 14. Define for $k \in \mathbb{Z}, x \in \mathbb{R}$, the fuzzy-wavelet-like operator

$$
\begin{aligned}
\left(R 1_{k} f\right)(x)= & \sum_{j=-\infty}^{\infty} 2 \varphi\left(2^{k} x-j\right) \\
& \odot(F R) \int_{0}^{1 / 2} f\left(\frac{t}{2^{k}}+\frac{j}{2^{k}}\right) d t .
\end{aligned}
$$

Then

$$
\begin{gathered}
D\left(\left(R 1_{k} f\right)(x), f(x)\right) \leq 2 \omega_{\mathbb{R}}\left(f, \frac{2 a+1}{2^{k+1}}\right), \\
D^{*}\left(\left(R 1_{k} f\right), f\right) \leq 2 \omega_{\mathbb{R}}\left(f, \frac{2 a+1}{2^{k+1}}\right),
\end{gathered}
$$

for all $k \in \mathbb{Z}, x \in \mathbb{R}$. When $f \in C_{F}^{U}(\mathbb{R})$ then as $k \rightarrow+\infty$ one gets $\omega_{\mathbb{R}}\left(f,(2 a+1) / 2^{k+1}\right) \rightarrow 0$ and $\lim _{k \rightarrow+\infty} R 1_{k} f=f$.

Proof. We want to estimate

$$
\begin{aligned}
& D\left(\left(R 1_{k} f\right)(x), f(x)\right) \\
& =D\left(\sum_{j}^{*} 2 \varphi\left(2^{k} x-j\right)(F R)^{2 \varphi\left(2^{k} x-j\right)(F R)}\right. \\
& \left.\odot \int_{0}^{1 / 2} f\left(\frac{t}{2^{k}}+\frac{j}{2^{k}}\right) d t, f(x) \odot 1\right)
\end{aligned}
$$

$$
\begin{aligned}
& =D\left(\sum_{\substack{j \\
k_{x-j \in[-a, a]}}}^{*} 2 \varphi\left(2^{k} x-j\right)\right. \\
& \odot(F R) \int_{0}^{1 / 2} f\left(\frac{t}{2^{k}}+\frac{j}{2^{k}}\right) d t \\
& \left.\sum_{j}^{*} 2 \varphi\left(2^{k} x-j\right)\right) \odot(F R) \int_{0}^{1 / 2} f(x) d t
\end{aligned}
$$$$
\leq \sum_{\substack{j \\{ }_{2} k_{x-j \in[-a, a]}}} D\left(2 \varphi\left(2^{k} x-j\right) \odot(F R) \int_{0}^{1 / 2} f\left(\frac{t}{2^{k}}+\frac{j}{2^{k}}\right) d t\right.
$$

$$
\left.2 \varphi\left(2^{k} x-j\right) \odot(F R) \int_{0}^{1 / 2} f(x) d t\right)
$$

$$
\begin{aligned}
& =2 \sum_{\substack{j \\
{ }_{2} k_{x-j \in[-a, a]}}} \varphi\left(2^{k} x-j\right) \\
& \quad \times \int_{0}^{1 / 2} D\left(f\left(\frac{t}{2^{k}}+\frac{j}{2^{k}}\right), f(x)\right) d t=:(*) .
\end{aligned}
$$


Clearly, we have

$$
\begin{aligned}
\frac{-a}{2^{k}}<\frac{j}{2^{k}}-x<\frac{a}{2^{k}} \longrightarrow \frac{t}{2^{k}}+\frac{j}{2^{k}}-x & <\frac{2 a+1}{2^{k+1}}, \\
0 & <t<\frac{1}{2} .
\end{aligned}
$$

Therefore, we conclude that

$$
\begin{aligned}
(*) \leq & 2 \sum_{{ }_{2} k_{x-j \in[-a, a]}} \varphi\left(2^{k} x-j\right) \omega_{\mathbb{R}} \\
& \times\left(f, \frac{2 a+1}{2^{k+1}}\right)=2 \omega_{\mathbb{R}}\left(f, \frac{2 a+1}{2^{k+1}}\right),
\end{aligned}
$$

and hence

$$
D^{*}\left(\left(R 1_{K} f\right), f\right) \leq 2 \omega_{\mathbb{R}}\left(f, \frac{2 a+1}{2^{k+1}}\right) .
$$

Theorem 16. All assumptions here are as in Theorem 14. Define for $k \in \mathbb{Z}, x \in \mathbb{R}, n \geq 2$ the fuzzy-wavelet-like operator

$$
\begin{aligned}
\left(R 2_{k} f\right)(x)= & \sum_{j=-\infty}^{\infty} 2 \odot(F R) \\
& \times \int_{0}^{1 / 2} f\left(\frac{t}{2^{k+n}}+\frac{j}{2^{k}}\right) d t \odot \varphi\left(2^{k} x-j\right) .
\end{aligned}
$$

Then

$$
\begin{gathered}
D\left(\left(R 2_{k} f\right)(x), f(x)\right) \leq 2 \omega_{\mathbb{R}}\left(f, \frac{2^{n+1} a+1}{2^{k+n+1}}\right), \\
D^{*}\left(\left(R 2_{k} f\right), f\right) \leq 2 \omega_{\mathbb{R}}\left(f, \frac{2^{n+1} a+1}{2^{k+n+1}}\right),
\end{gathered}
$$

when $f \in C_{F}^{U}(\mathbb{R})$ then as $k \rightarrow+\infty$ one gets $\omega_{\mathbb{R}}\left(f,\left(2^{n+1} a+\right.\right.$ $\left.1) /\left(2^{k+n+1}\right)\right) \rightarrow 0$ and $\lim _{k \rightarrow+\infty} R 2_{k} f=f$.

Proof. We need to estimate

$$
\begin{aligned}
& D\left(\left(R 2_{k} f\right)(x), f(x)\right) \\
& =D\left(\sum_{{ }_{2} k_{x-j \in[-a, a]}}^{*} 2 \odot(F R) \int_{0}^{1 / 2} f\left(\frac{t}{2^{k+n}}+\frac{j}{2^{k}}\right) d t\right. \\
& \left.\odot \varphi\left(2^{k} x-j\right), f(x) \odot 1\right)
\end{aligned}
$$

$$
\begin{aligned}
& =D\left(\sum_{j}^{*} 2 \odot(F R)\right. \\
& \quad \times \int_{0}^{1 / 2} f\left(\frac{t}{2^{k+n}}+\frac{j}{2^{k}}\right) d t \odot \varphi\left(2^{k} x-j\right),
\end{aligned}
$$$$
\left.\sum_{\substack{j \\{ }_{2} k_{x-j \in[-a, a]}}}^{*} \odot(F R) \int_{0}^{1 / 2} f(x) d t \odot \varphi\left(2^{k} x-j\right)\right)
$$$$
\leq \sum_{\substack{j \\ 2^{k}-j \in[-a, a]}}^{*} 2 D\left((F R) \int_{0}^{1 / 2} f\left(\frac{t}{2^{k+n}}+\frac{j}{2^{k}}\right) d t \odot \varphi\left(2^{k} x-j\right),\right.
$$

$$
\left.\sum_{\substack{j \\{ }_{2} k_{x-j \in[-a, a]}}}^{*}(F R) \int_{0}^{1 / 2} f(x) d t \odot \varphi\left(2^{k} x-j\right)\right)
$$

$$
\begin{aligned}
& \leq 2 \sum_{j}{ }_{2}{ }_{k_{x-j \in[-a, a]}}^{*} \varphi\left(2^{k} x-j\right) \\
& \quad \times \int_{0}^{1 / 2} D\left(f\left(\frac{t}{2^{k+n}}+\frac{j}{2^{k}}\right), f(x)\right) d t=:(* *) .
\end{aligned}
$$

Here, we have

$$
\begin{aligned}
-\frac{a}{2^{k}}<\frac{j}{2^{k}}-x<\frac{a}{2^{k}} \Longrightarrow \frac{t}{2^{k}}+\frac{j}{2^{k}}-x<\frac{2^{n+1} a+1}{2^{k+n+1}}, \\
0<t<\frac{1}{2} .
\end{aligned}
$$

Now, by using the above inequality in $(* *)$, we can write

$$
\begin{aligned}
(* *) \leq & 2 \sum_{{ }_{2} k_{x-j \in[-a, a]}} \varphi\left(2^{k} x-j\right) \omega_{\mathbb{R}} \\
& \times\left(f, \frac{2^{n+1} a+1}{2^{k+n+1}}\right)=2 \omega_{\mathbb{R}}\left(f, \frac{2^{n+1} a+1}{2^{k+n+1}}\right),
\end{aligned}
$$

and hence

$$
D^{*}\left(\left(R 2_{K} f\right), f\right) \leq 2 \omega_{\mathbb{R}}\left(f, \frac{2^{n+1} a+1}{2^{k+n+1}}\right) .
$$

Theorem 17. All assumptions here are as in Theorem 14. Define for $k \in \mathbb{Z}, x \in \mathbb{R}$ the fuzzy-wavelet-like operator

$$
\left(R 3_{K} f\right)(x):=\sum_{j=-\infty}^{\infty}{ }^{*} \delta_{k j}(f) \odot \varphi\left(2^{k} x-j\right),
$$


where

$$
\begin{aligned}
\delta_{k j}(f):= & \sum_{\widetilde{s}=0}^{n} \sum_{\widetilde{r}=0}^{n} \omega_{\widetilde{r}} \omega_{\widetilde{s}} \odot f\left(\frac{j}{2^{k}}+\frac{\widetilde{r}}{n 2^{k}}+\frac{\widetilde{s}}{n 2^{k}}\right), \quad n \in \mathbb{N}, \\
& \omega_{\tilde{r}}, \omega_{\widetilde{s}} \geq 0, \quad \sum_{\widetilde{r}=0}^{n} \omega_{\widetilde{r}}=1, \quad \sum_{\widetilde{s}=0}^{n} \omega_{\widetilde{s}}=1 .
\end{aligned}
$$

Then

$$
\begin{gathered}
D\left(\left(R 3_{k} f\right)(x), f(x)\right) \leq \omega_{\mathbb{R}}\left(f, \frac{a+2}{2^{k}}\right), \\
D^{*}\left(R 3_{k}, f\right) \leq \omega_{\mathbb{R}}\left(f, \frac{a+2}{2^{k}}\right), \quad \text { all } k \in \mathbb{Z}, x \in \mathbb{R} .
\end{gathered}
$$

When $f \in C_{F}^{U}(\mathbb{R})$ then as $k \rightarrow+\infty$ one gets $\omega_{\mathbb{R}}(f,(a+$ 2) $\left./ 2^{k}\right) \rightarrow 0$ and $\lim _{k \rightarrow+\infty} R 3_{k} f=f$.

Proof. We have the following:

$$
\begin{aligned}
D\left(\sum_{j=-\infty}^{\infty} \delta_{k j}(f) \odot \varphi\left(2^{k} x-j\right), f(x)\right) \\
=D\left(\sum_{2^{k} x-j \in[-a, a]}^{*} \delta_{k j}(f) \odot \varphi\left(2^{k} x-j\right),\right. \\
\left.\sum_{2^{k} x-j \in[-a, a]}^{*} f(x) \odot \varphi\left(2^{k} x-j\right)\right) \\
\leq \sum_{j} \varphi\left(2^{k} x-j\right) D\left(\delta_{k j}(f), f(x)\right) \\
=\sum_{j} \varphi\left(2^{k} x-j\right) \\
\quad \times D\left(\sum_{\tilde{s}=0}^{n} \sum_{\tilde{r}=0}^{n} w_{\tilde{r}} w_{\widetilde{s}} f\left(\frac{j}{2^{k}}+\frac{\tilde{r}}{n 2^{k}}+\frac{\widetilde{s}}{n 2^{k}}\right),\right. \\
\leq \sum_{j}^{n} \varphi\left(2^{k} x-j\right) \sum_{\tilde{s}=0}^{n} \sum_{\tilde{r}=0}^{n} w_{\tilde{r}} w_{\widetilde{s}} D \\
\quad \times\left(f\left(\frac{j}{2^{k}}+\frac{\tilde{r}}{n 2^{k}}+\frac{\widetilde{s}}{n 2^{k}}\right), f(x)\right) \leq \omega_{\mathbb{R}}\left(f, \frac{a+2}{2^{k}}\right) .
\end{aligned}
$$

Notice that

$$
\begin{aligned}
-\frac{a}{2^{k}}<\frac{j}{2^{k}}-x & <\frac{a}{2^{k}}, \quad 0<\frac{\widetilde{r}}{2^{k} n}<\frac{a}{2^{k}}, \\
0 & <\frac{\widetilde{s}}{2^{k} n}<\frac{1}{2^{k}} .
\end{aligned}
$$

Therefore,

$$
\left|\frac{j}{2^{k}}+\frac{\widetilde{r}}{2^{k} n}+\frac{\widetilde{s}}{2^{k} n}-x\right|<\frac{a+2}{2^{k}} .
$$

This completes the proof.

Theorem 18. Let $f \in C_{F}\left(\mathbb{R}^{2}\right)$ and the scaling function

$$
\phi(x, y)=\varphi(x) \varphi(y)
$$

a real-valued bounded function with $\operatorname{supp} \varphi(x, y) \subseteq[-a, a] \times$ $[-b, b], 0<a, b<+\infty, \varphi(x, y) \geq 0$, such that $\sum_{i=-\infty}^{\infty} \sum_{j=-\infty}^{\infty} \varphi(x-i) \varphi(y-j)=1$ on $\mathbb{R}^{2}$. For $k \in \mathbb{Z}, x \in \mathbb{R}$, put

$$
\begin{aligned}
\left(2 D G_{K} f\right)(x, y):= & \sum_{j=-\infty}^{\infty} \sum_{j=-\infty}^{\infty} f\left(\frac{i}{2^{k}}, \frac{j}{2^{k}}\right) \\
& \odot \varphi\left(2^{k} x-i\right) \varphi\left(2^{k} y-j\right),
\end{aligned}
$$

which is a fuzzy-wavelet-like operator. Then

$$
\begin{aligned}
& D\left(\left(2 D G_{k} f\right)(x, y), f(x, y)\right) \leq \omega_{\mathbb{R}^{2}}\left(f, \frac{\sqrt{a^{2}+b^{2}}}{2^{k}}\right), \\
& D^{*}\left(2 D G_{k} f(x, y), f(x, y)\right) \leq \omega_{\mathbb{R}^{2}}\left(f, \frac{\sqrt{a^{2}+b^{2}}}{2^{k}}\right),
\end{aligned}
$$

for all $x \in \mathbb{R}$ and $k \in \mathbb{Z}$. If $f \in C_{F}^{U}\left(\mathbb{R}^{2}\right)$, then as $k \rightarrow+\infty w$ one gets $\omega_{\mathbb{R}^{2}}\left(f,\left(\sqrt{a^{2}+b^{2}}\right) / 2^{k}\right) \rightarrow 0$ and $\lim _{k \rightarrow+\infty} 2 D G_{k} f=$ $f$, pointwise and uniformly with rates.

Proof. We have the following:

$$
\begin{aligned}
& D\left(\left(2 D G_{K} f\right)(x, y), f(x, y)\right) \\
& =D\left(\sum_{i=-\infty}^{\infty} \sum_{j=-\infty}^{\infty}{ }^{*} f\left(\frac{i}{2^{k}}, \frac{j}{2^{k}}\right)\right. \\
& \odot \varphi\left(2^{k} x-i\right) \varphi\left(2^{k} y-j\right), \\
& \left.\sum_{i=-\infty}^{\infty} \sum_{j=-\infty}^{\infty} \varphi\left(2^{k} x-i\right) \varphi\left(2^{k} y-j\right) \odot f(x, y)\right) \\
& \leq \sum_{i=-\infty}^{\infty} \sum_{j=-\infty}^{\infty} \varphi\left(2^{k} x-i\right) \varphi\left(2^{k} y-j\right) \\
& \quad \times D\left(f\left(\frac{i}{2^{k}}, \frac{j}{2^{k}}\right), f(x, y)\right)=:(* * *) .
\end{aligned}
$$

According to this fact that

$$
\frac{i-a}{2^{k}}<x<\frac{i+a}{2^{k}}, \quad \frac{j-b}{2^{k}}<y<\frac{j+b}{2^{k}},
$$

we conclude that

$$
\left|\frac{i}{2^{k}}-x\right| \leq \frac{a}{2^{k}}, \quad\left|\frac{j}{2^{k}}-y\right| \leq \frac{b}{2^{k}},
$$


and hence

$$
\begin{aligned}
&(* * *) \leq \sum_{i} \sum_{j} \varphi\left(2^{k} x-i\right) \varphi\left(2^{k} y-j\right) \omega_{\mathbb{R}^{2}} \\
& \quad \times\left(f, \frac{\sqrt{a^{2}+b^{2}}}{2^{k}}\right)=\omega_{\mathbb{R}^{2}}\left(f, \frac{\sqrt{a^{2}+b^{2}}}{2^{k}}\right), \\
& D^{*}\left(\left(2 D G_{K} f\right)(x, y), f(x, y)\right) \leq \omega_{\mathbb{R}^{2}}\left(f, \frac{\sqrt{a^{2}+b^{2}}}{2^{k}}\right) .
\end{aligned}
$$

This completes the proof.

Theorem 19. All assumptions here are as in Theorem 18. For $k \in \mathbb{Z}, x \in \mathbb{R}$, put

$$
\begin{aligned}
\left(2 D G 1_{K} f\right)(x, y) & \\
=\sum_{i=-\infty}^{\infty} \sum_{j=-\infty}^{\infty}( & 2^{2 k} \odot(F R) \\
& \times \int_{0}^{2^{-k}} \int_{0}^{2^{-k}} f\left(t+\frac{i}{2^{k}}, s+\frac{j}{2^{k}}\right) d t d s \\
& \left.\odot \varphi\left(2^{k} x-i\right) \varphi\left(2^{k} y-j\right)\right)
\end{aligned}
$$

which is a fuzzy-wavelet-like operator. Then

$$
\begin{aligned}
& D\left(\left(2 D G 1_{k} f\right)(x, y), f(x, y)\right) \\
& \leq \omega_{\mathbb{R}^{2}}\left(f, \frac{\sqrt{(a+1)^{2}+(b+1)^{2}}}{2^{k}}\right), \\
& D^{*}\left(2 D G 1_{k} f(x, y), f(x, y)\right) \\
& \leq \omega_{\mathbb{R}^{2}}\left(f, \frac{\sqrt{(a+1)^{2}+(b+1)^{2}}}{2^{k}}\right),
\end{aligned}
$$

for all $x \in \mathbb{R}$ and $k \in \mathbb{Z}$. If $f \in C_{F}^{U}\left(\mathbb{R}^{2}\right)$, then as $k \rightarrow+\infty w$ one gets

$$
\omega_{\mathbb{R}^{2}}\left(f, \frac{\sqrt{(a+1)^{2}+(b+1)^{2}}}{2^{k}}\right) \rightarrow 0
$$

and $\lim _{k \rightarrow+\infty} 2 D G 1_{k} f=f$, pointwise and uniformly with rates.
Proof. We have the following:

$$
\begin{aligned}
& D\left(\left(2 D G 1_{K} f\right)(x, y), f(x, y)\right) \\
& =D\left(\sum_{i=-\infty}^{\infty} \sum_{j=-\infty}^{\infty} 2^{2 k}\right. \\
& \odot(F R) \int_{0}^{2^{-k}} \int_{0}^{2^{-k}} f\left(t+\frac{i}{2^{k}}, s+\frac{j}{2^{k}}\right) d t d s \\
& \odot \varphi\left(2^{k} x-i\right) \varphi\left(2^{k} y-j\right) \text {, } \\
& \sum_{i=-\infty}^{\infty} \sum_{j=-\infty}^{\infty} \varphi\left(2^{k} x-i\right) \varphi\left(2^{k} y-j\right) 2^{2 k} \\
& \left.\odot(F R) \int_{0}^{2^{-k}} \int_{0}^{2^{-k}} f(x, y) d t d s\right) \\
& \leq 2^{2 k} \sum_{i=-\infty}^{\infty} \sum_{j=-\infty}^{\infty} \varphi\left(2^{k} x-i\right) \varphi\left(2^{k} y-j\right) \\
& \times D\left((F R) \int_{0}^{2^{-k}} \int_{0}^{2^{-k}} f\left(t+\frac{i}{2^{k}}, s+\frac{j}{2^{k}}\right),\right. \\
& \left.(F R) \int_{0}^{2^{-k}} \int_{0}^{2^{-k}} f(x, y) d t d s\right) \\
& \leq 2^{2 k} \sum_{i=-\infty}^{\infty} \sum_{j=-\infty}^{\infty} \varphi\left(2^{k} x-i\right) \varphi\left(2^{k} y-j\right) \\
& \times \int_{0}^{2^{-k}} \int_{0}^{2^{-k}} D\left(f\left(t+\frac{i}{2^{k}}, s+\frac{j}{2^{k}}\right),\right. \\
& f(x, y) d t d s)=:(* * * *) .
\end{aligned}
$$

According to this fact that

$$
\begin{gathered}
0 \leq t \leq \frac{1}{2^{k}}, \quad 0 \leq s \leq \frac{1}{2^{k}}, \\
\frac{i-a}{2^{k}}<x<\frac{i+a}{2^{k}}, \quad \frac{j-b}{2^{k}}<y<\frac{j+b}{2^{k}},
\end{gathered}
$$

we conclude that

$$
\left|t+\frac{i}{2^{k}}-x\right| \leq \frac{a+1}{2^{k}}, \quad\left|s+\frac{j}{2^{k}}-y\right| \leq \frac{b+1}{2^{k}},
$$

and hence

$$
\begin{aligned}
(* * * *) \leq & 2^{2 k} \sum_{i} \sum_{j} \varphi\left(2^{k} x-i\right) \varphi\left(2^{k} y-j\right) \omega_{\mathbb{R}^{2}} \\
& \times\left(f, \frac{\sqrt{(a+1)^{2}+(b+1)^{2}}}{2^{k}}\right) 2^{-2 k} \\
= & \omega_{\mathbb{R}^{2}}\left(f, \frac{\sqrt{(a+1)^{2}+(b+1)^{2}}}{2^{k}}\right),
\end{aligned}
$$




$$
\begin{aligned}
& D^{*}\left(\left(2 D G 1_{K} f\right)(x, y), f(x, y)\right) \\
& \quad \leq \omega_{\mathbb{R}^{2}}\left(f, \frac{\sqrt{(a+1)^{2}+(b+1)^{2}}}{2^{k}}\right) .
\end{aligned}
$$

This completes the proof.

Theorem 20. Let $f: \mathbb{R} \rightarrow \mathbb{R}_{F}$ and the scaling function $\varphi(x)$ a real-valued bounded function with $\operatorname{supp} \varphi \subseteq[-a, a], 0<a<$ $+\infty$, such that

(1) $\sum_{j=-\infty}^{\infty} \varphi(x-j)=1$ on $\mathbb{R}$,

(2) there exists $a, b \in \mathbb{R}$ such that $\varphi$ is nondecreasing for $x \leq a$ and $\varphi$ is nonincreasing for $x \geq b$

(the above imply $\varphi \geq 0$ ). Let $f(x, y)$ be nondecreasing fuzzy function, then $\left(R 3_{k} f\right)(x)$ is nondecreasing fuzzy valued functions for any $k \in \mathbb{Z}$.

Proof. The proof is similar to the proof of [15, Theorem 12.14]. Let $x_{n}, x \in \mathbb{R}$ such that $x_{n} \rightarrow x$, as $n \rightarrow+\infty$, then $D\left(f\left(x_{n}\right), f(x)\right) \rightarrow 0$, by fuzzy continuity of $f$. But we have

$$
\begin{aligned}
D\left(f\left(x_{n}\right), f(x)\right)=\sup _{r \in[0,1]} \max \{ & \left|\left(f\left(x_{n}\right)\right)_{-}^{(r)}-(f(x))_{-}^{(r)}\right|, \\
& \left.\left|\left(f\left(x_{n}\right)\right)_{+}^{(r)}-(f(x))_{+}^{(r)}\right|\right\} .
\end{aligned}
$$

That is, $\left|\left(f\left(x_{n}\right)\right)_{ \pm}^{(r)}-(f(x))_{ \pm}^{(r)}\right| \rightarrow 0$, for all $0 \leq r \leq 1$ as $n \rightarrow+\infty$, respectively. Therefore $(f)_{+}^{(r)} \in C(\mathbb{R})$, for all $0 \leq$ $r \leq 1$, that is, real-valued continuous functions on $\mathbb{R}$. Since $f$ is fuzzy nondecreasing by Definition 11 we get that $(f)_{ \pm}^{(r)}$ are non-decreasing, for all $r \in[0,1]$, respectively.

Next we observe that

$$
\begin{aligned}
{\left[\left(R 3_{k} f\right)(x)\right]^{r}=} & \sum_{j=-\infty}^{\infty} \sum_{\tilde{s}=0}^{n} \sum_{\tilde{r}=0}^{n} w_{\tilde{r}} w_{\tilde{s}} \\
& \times\left[f\left(\frac{j}{2^{k}}+\frac{\widetilde{r}}{n 2^{k}}+\frac{\widetilde{s}}{n 2^{k}}\right)\right]^{r} \varphi\left(2^{k} x-j\right) .
\end{aligned}
$$

That is,

$$
\begin{array}{r}
{\left[\left(\left(R 3_{k} f\right)(x)\right)_{-}^{(r)},\left(\left(R 3_{k} f\right)(x)\right)_{+}^{(r)}\right]} \\
=\sum_{j=-\infty}^{\infty}\left(\sum_{\tilde{s}=0}^{n} \sum_{\tilde{r}=0}^{n} w_{\tilde{r}} w_{\tilde{s}}\left(f\left(\frac{j}{2^{k}}+\frac{\widetilde{r}}{n 2^{k}}+\frac{\widetilde{s}}{n 2^{k}}\right)\right)_{-}^{(r)},\right. \\
\\
\left.\sum_{\tilde{s}=0}^{n} \sum_{\tilde{r}=0}^{n} w_{\widetilde{r}} w_{\widetilde{s}}\left(f\left(\frac{j}{2^{k}}+\frac{\tilde{r}}{n 2^{k}}+\frac{\widetilde{s}}{n 2^{k}}\right)\right)_{+}^{(r)}\right)
\end{array}
$$

$$
\begin{aligned}
& \times \varphi\left(2^{k} x-j\right) \\
&= \sum_{j=-\infty}^{\infty} \sum_{\tilde{s}=0}^{n} \sum_{\tilde{r}=0}^{n} w_{\tilde{r}} w_{\widetilde{s}}\left(f\left(\frac{j}{2^{k}}+\frac{\tilde{r}}{n 2^{k}}+\frac{\widetilde{s}}{n 2^{k}}\right)\right)_{-}^{(r)} \\
& \times \varphi\left(2^{k} x-j\right), \\
& \sum_{j=-\infty}^{\infty} \sum_{\widetilde{s}=0}^{n} \sum_{\widetilde{r}=0}^{n} w_{\tilde{r}} w_{\widetilde{s}}\left(f\left(\frac{j}{2^{k}}+\frac{\tilde{r}}{n 2^{k}}+\frac{\widetilde{s}}{n 2^{k}}\right)\right)_{+}^{(r)} \varphi\left(2^{k} x-j\right) \\
&=\left[\left(E_{k}(f)_{-}^{(r)}\right)(x),\left(E_{k}(f)_{+}^{(r)}\right)(x)\right] .
\end{aligned}
$$

So whenever $x_{1} \leq x_{2}$ we get

$$
\left(R 3_{k}(f)_{ \pm}^{(r)}\right)\left(x_{1}\right) \leq\left(R 3_{k}(f)_{ \pm}^{(r)}\right)\left(x_{2}\right), \quad \forall r \in[0,1]
$$

Therefore, $R 3_{k} f$ is nondecreasing.

Theorem 21. Let $f$ and $\varphi$ be as in Theorem 20. Then $\left(R 1_{k} f\right)(x)$ is a nondecreasing fuzzy valued function for any $k \in \mathbb{Z}$.

Proof. The proof is similar to the proof of Theorem 20.

\section{Numerical Examples}

In this section, we present two examples. Also, we apply the following scaling function [1]:

$$
\varphi(x)= \begin{cases}1 & \frac{-1}{2} \leq x \leq \frac{1}{2}, \\ 0 & \text { elsewhere. }\end{cases}
$$

Example 1. In this example, we want to apply the fuzzywavelet-like operator $R 1_{k} f$ defined in Theorem 15 to approximate a given function $f(x)=e^{u x}$, where $[u]^{r}=[1+r, 3-r]$, $r \in[0,1]$. To do this, we consider $k=6$. Comparing the numerical result with $f(x)$ is done in Figure 1.

Example 2. Consider $f(x, y)=e^{u x y}$, where $[u]^{r}=[r, 2-$ $r], r \in[0,1]$. Now, we apply the fuzzy- wavelet-like operator $2 D G_{k} f$ defined in Theorem 18 to approximate $f(x, y)$. We also compare the result of using $2 D G_{k} f$ with $f(x, y)$ in $x=$ $0.9, y=0.9$. For more details, see Figures 2 and 3 .

\section{An Application}

In this section, we approximate the integration of continuous fuzzy real number valued function of two variables by using the fuzzy-wavelet-like operator $2 D G_{k} f$ defined in Theorem 18. Consider the following fuzzy integral:

$$
(F R) \int_{-1}^{1} \int_{-1}^{1} f(x, y) d x d y
$$


By using the fuzzy-wavelet-like operator $2 D G_{k} f$, we get

$$
\begin{aligned}
(F R) & \int_{-1}^{1} \int_{-1}^{1} f(x, y) d x d y \\
\cong & (F R) \int_{-1}^{1} \int_{-1}^{1}\left(2 D G_{K} f\right)(x, y) d x d y \\
= & (F R) \int_{-1}^{1} \int_{-1}^{1} \sum_{i=-\infty}^{\infty} \sum_{j=-\infty}^{\infty} f\left(\frac{i}{2^{k}}, \frac{j}{2^{k}}\right) \\
= & \sum_{i=-\infty}^{\infty} \sum_{j=-\infty}^{\infty} f\left(\frac{i}{2^{k}}, \frac{j}{2^{k}}\right) \\
& \odot \int_{-1}^{1} \varphi\left(2^{k} x-i\right) d x \int_{-1}^{1} \varphi\left(2^{k} y-j\right) d y \\
= & \sum_{i=-\infty}^{\infty} 2^{*} \sum_{j=-\infty}^{\infty} f\left(\frac{i}{2^{k}}, \frac{j}{2^{k}}\right) d x d y \\
= & \sum_{i=-2^{k}}^{*} \sum_{j=-2^{k}}^{*} f\left(\frac{i}{2^{k}}, \frac{j}{2^{k}}\right) \odot\left(\frac{1}{2^{k}} \int_{-1 / 2}^{1 / 2} \varphi(u) d u\right)^{2} \\
& \left.\odot \int_{-2^{k}-i}^{2^{k}-i} \varphi(u) d u\right)\left(\frac{1}{2^{k}} \int_{-2^{k}-j}^{2^{k}-j} \varphi(u) d u\right)^{*}
\end{aligned}
$$

So, we have

$$
(F R) \int_{-1}^{1} \int_{-1}^{1} f(x, y) d x d y \cong \frac{1}{2^{2 k}} \odot \sum_{i=-2^{k}}^{2^{k}-1} \sum_{j=-2^{k}}^{*} f\left(\frac{i}{2^{k}}, \frac{j}{2^{k}}\right) .
$$

Theorem 22. Let $f \in C_{F}\left([a, b]^{2}\right)$. Then

$$
\begin{aligned}
& D\left((F R) \int_{-1}^{1} \int_{-1}^{1} f(x, y) d x d y\right. \\
& \left.\quad \frac{1}{2^{2 k}} \odot \sum_{i=-2^{k}}^{2^{k}-1} \sum_{j=-2^{k}}^{*}\left(\frac{i}{2^{k}}, \frac{j}{2^{k}}\right)\right) \\
& \leq 4 \omega_{[-1,1]^{2}}\left(f, \frac{\sqrt{2}}{2^{k}}\right) .
\end{aligned}
$$

Proof. We have the following:

$$
\begin{aligned}
& D\left((F R) \int_{-1}^{1} \int_{-1}^{1} f(x, y) d x d y, \frac{1}{2^{2 k}} \odot \sum_{i=-2^{k}}^{2^{k}-1^{*}} \sum_{j=-2^{k}}^{2^{k}-1} f\left(\frac{i}{2^{k}}, \frac{j}{2^{k}}\right)\right) \\
& =D\left(\sum_{i=-2^{k}}^{2^{k}-1} \sum_{j=-2^{k}}^{2^{k}-1^{*}}(F R) \int_{j / 2^{k}}^{(j+1) / 2^{k}} \int_{i / 2^{k}}^{(i+1) / 2^{k}} f(x, y) d x d y\right. \\
& \left.\sum_{i=-2^{k}}^{2^{k}-1} \sum_{j=-2^{k}}^{2^{k}-1^{*}}(F R) \int_{j / 2^{k}}^{(j+1) / 2^{k}} \int_{i / 2^{k}}^{(i+1) / 2^{k}} f\left(\frac{i}{2^{k}}, \frac{j}{2^{k}}\right) d x d y\right) \\
& \leq \sum_{i=-2^{k}}^{2^{k}-1^{*}} \sum_{j=-2^{k}}^{2^{k}-1} \int_{j / 2^{k}}^{(j+1) / 2^{k}} \int_{i / 2^{k}}^{(i+1) / 2^{k}} D\left(f(x, y), f\left(\frac{i}{2^{k}}, \frac{j}{2^{k}}\right)\right) d x d y \\
& \left.\leq \sum_{i=-2^{k}}^{2^{k}-1} \sum_{j=-2^{k}}^{*} \int_{i / 2^{k}}^{2^{k}-1} \int_{j / 2^{k}}^{(i+1) / 2^{k}} \omega_{[-1,1]^{2}}^{(j+1) / 2^{k}} f, \sqrt{\left(x-\frac{i}{2^{k}}\right)^{2}+\left(y-\frac{j}{2^{k}}\right)^{2}}\right) d x d y \\
& \leq \sum_{i=-2^{k}}^{2^{k}-1^{*}} \sum_{j=-2^{k}}^{2^{k}-1} \int_{i / 2^{k}}^{(i+1) / 2^{k}} \int_{j / 2^{k}}^{(j+1) / 2^{k}} \omega_{[-1,1]^{2}}\left(f, \frac{\sqrt{2}}{2^{k}}\right) d x d y \\
& =\sum_{i=-2^{k}}^{2^{k}-1} \sum_{j=-2^{k}}^{*} \frac{1}{2^{k}-1} \omega_{[-1,1]^{2}}\left(f, \frac{\sqrt{2}}{2^{k}}\right)=4 \omega_{[-1,1]^{2}}\left(f, \frac{\sqrt{2}}{2^{k}}\right) .
\end{aligned}
$$




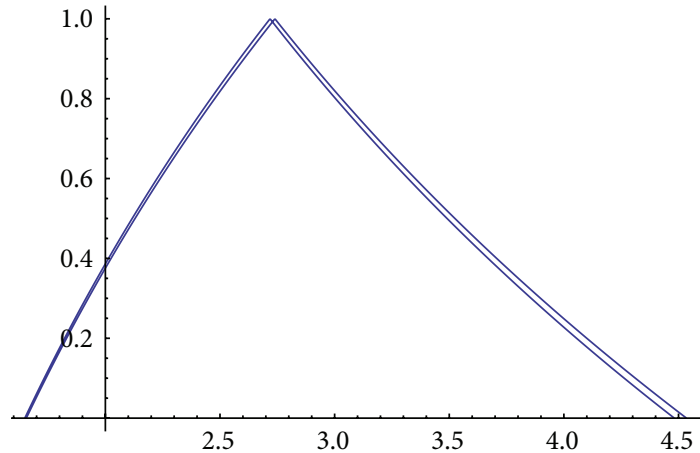

FIGURE 1: Comparing $f(x)$ and fuzzy-wavelet-like operator $R 1_{k} f$ with $k=6$ in $x=0.5$.

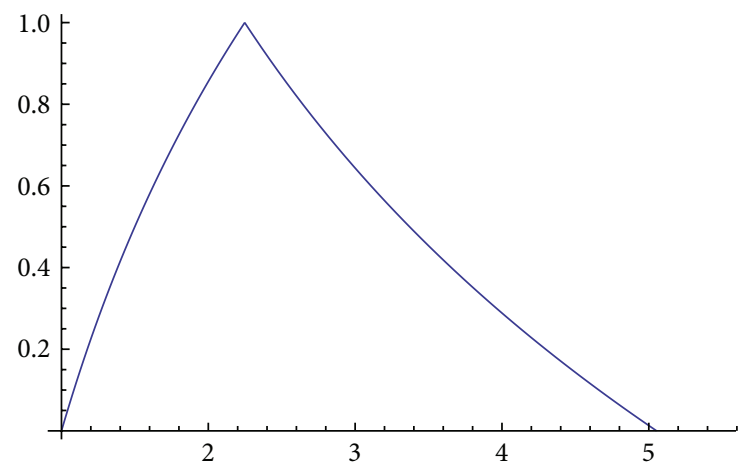

Figure 2: The fuzzy number $f(x, y)$ in $x=0.9, y=0.9$.

Example 3. Consider the following fuzzy integral:

$$
\text { (FR) } \int_{-1}^{1} \int_{-1}^{1} e^{u x-y} d x d y,
$$

where $u=(1+r, 3-r)$. The exact solution of this example is $\left(I_{1}(r), I_{2}(r)\right), 0 \leq r \leq 1$, where

$$
\begin{aligned}
& I_{1}(r)=\left(\frac{1-e^{r-3}}{3-r}+\frac{1}{r+1}\left(e^{r+1}-1\right)\right)\left(e-e^{-1}\right), \\
& I_{2}(r)=\left(\frac{1-e^{-(r+1)}}{r+1}+\frac{1}{3-r}\left(e^{3-r}-1\right)\right)\left(e-e^{-1}\right) .
\end{aligned}
$$

Let $2 D G_{k} f=\left(2 D G_{k} f(r), \overline{2 D G_{k} f}(r)\right), 0 \leq r \leq 1$. By using proposed method in (53), we present approximate solution to this example for different values of $k$. To compare the exact and the approximate solutions, see Tables 1,2, and 3.

\section{Conclusions}

To approximate the continuous fuzzy functions of one and two variables, some new fuzzy-wavelet-like operators via a real-valued scaling function are defined. Convergence of defined fuzzy like operators is investigated by using the

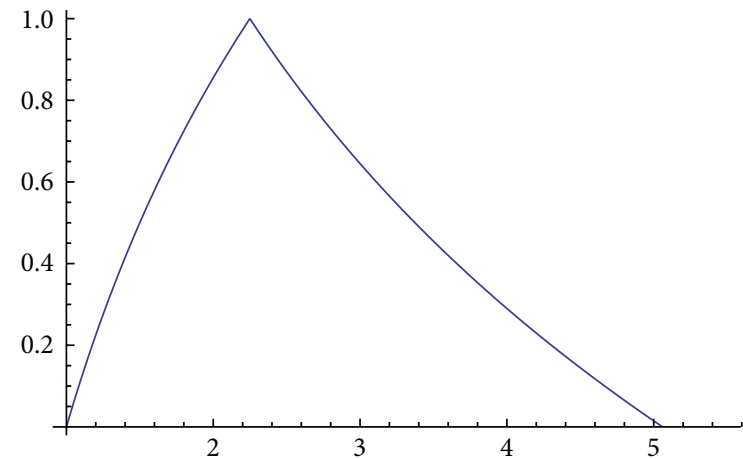

FIGURE 3: Approximation of $f(x, y)$ by using fuzzy wavelet like operator $2 D G_{k} f$ with $k=10$ in $x=0.9, y=0.9$.

TABLE 1: Numerical results for Example 3 with $k=6$.

\begin{tabular}{lcc}
\hline$r$-level & $\left|2 D G_{k} f-I_{1}\right|$ & $\left|\overline{2 D G_{k} f}-I_{2}\right|$ \\
\hline 0.0 & 0.0570312 & 0.0892018 \\
0.1 & 0.0556607 & 0.0704432 \\
0.2 & 0.054025 & 0.0537745 \\
0.3 & 0.0520895 & 0.0389791 \\
0.4 & 0.0498158 & 0.0258617 \\
0.5 & 0.0471608 & 0.0142472 \\
0.6 & 0.0440764 & 0.0039777 \\
0.7 & 0.0405092 & 0.0050884 \\
0.8 & 0.0363992 & 0.0130785 \\
0.9 & 0.0316798 & 0.020107 \\
1.0 & 0.0262767 & 0.0262767 \\
\hline
\end{tabular}

TABLE 2: Numerical results for Example 3 with $k=8$.

\begin{tabular}{lcc}
\hline$r$-level & $\left|2 D G_{k} f-I_{1}\right|$ & $\left|\overline{2 D G_{k} f}-I_{2}\right|$ \\
\hline 0.0 & 0.0142312 & 0.0220079 \\
0.1 & 0.0138981 & 0.0173406 \\
0.2 & 0.0134993 & 0.0131953 \\
0.3 & 0.0130261 & 0.00951781 \\
0.4 & 0.012469 & 0.00625923 \\
0.5 & 0.0118171 & 0.00337565 \\
0.6 & 0.0110587 & 0.00082763 \\
0.7 & 0.0101801 & 0.00142031 \\
0.8 & 0.0916663 & 0.00340001 \\
0.9 & 0.00800153 & 0.00514009 \\
1.0 & 0.00666625 & 0.00666625 \\
\hline
\end{tabular}

modulus of continuity. To approximate the integration of continuous fuzzy real number valued function of two variables by using the fuzzy-wavelet-like operator $2 D G_{k} f$ defined in Theorem 18, an application is presented. 
TABLE 3: Numerical results for Example 3 with $k=10$.

\begin{tabular}{lcc}
\hline$r$-level & $\left|2 D G_{k} f-I_{1}\right|$ & $\left|\overline{2 D G_{k} f}-I_{2}\right|$ \\
\hline 0.0 & 0.00355617 & 0.00548333 \\
0.1 & 0.0034735 & 0.00431794 \\
0.2 & 0.00337443 & 0.00328303 \\
0.3 & 0.0032568 & 0.00236503 \\
0.4 & 0.00311822 & 0.000551771 \\
0.5 & 0.00295602 & 0.0008321 \\
0.6 & 0.00276719 & 0.000196327 \\
0.7 & 0.00254839 & 0.000364475 \\
0.8 & 0.0022959 & 0.000858269 \\
0.9 & 0.00200556 & 0.00129221 \\
1.0 & 0.00167272 & 0.00167272 \\
\hline
\end{tabular}

\section{References}

[1] G. A. Anastassiou, "Fuzzy wavelet type operators," Nonlinear Functional Analysis and Applications, vol. 9, no. 2, pp. 251-269, 2004.

[2] S. Abbasbandy and M. Amirfakhrian, "Approximation of fuzzy functions by distance method," Mathematics Scientific Journal, vol. 2, no. 4, pp. 1-12, 2004.

[3] S. Abbasbandy, "Interpolation of fuzzy data by complete splines," The Korean Journal of Computational \& Applied Mathematics, vol. 8, no. 3, pp. 587-594, 2001.

[4] S. Abbasbandy and E. Babolian, "Interpolation of fuzzy data by natural splines," The Korean Journal of Computational \& Applied Mathematics, vol. 5, no. 2, pp. 457-463, 1998.

[5] H. Behforooz, R. Ezzati, and S. Abbasbandy, "Interpolation of fuzzy data by using E(3) cubic splines," International Journal of Pure and Applied Mathematics, vol. 60, no. 4, pp. 383-392, 2010.

[6] S. Abbasbandy, R. Ezzati, and H. Behforooz, "Interpolation of fuzzy data by using fuzzy splines," International Journal of Uncertainty, Fuzziness and Knowledge-Based Systems, vol. 16, no. 1, pp. 107-115, 2008.

[7] S. Ziari, R. Ezzati, and S. Abbasbandy, "Numerical solution of linear fuzzy Fred-holm integral equations of the second kind using fuzzy Haar wavelet," Communications in Computer and Information Science, vol. 299, pp. 79-89, 2012.

[8] R. Goetschel, Jr. and W. Voxman, "Elementary fuzzy calculus," Fuzzy Sets and Systems, vol. 18, no. 1, pp. 31-43, 1986.

[9] M. Friedman, M. Ming, and A. Kandel, "Numerical methods for calculating the fuzzy integral," Fuzzy Sets and Systems, vol. 83, no. 1, pp. 57-62, 1996.

[10] S. G. Gal, "Approximation theory in fuzzy setting," in Handbook of Analytic-Computational Methods in Applied Mathematics, G. Anastassiou, Ed., pp. 617-666, Chapman \& Hall/CRC, Boca Raton, Fla, USA, 2000.

[11] C. Wu and Z. Gong, "On Henstock integral of fuzzy-numbervalued functions. I," Fuzzy Sets and Systems, vol. 120, no. 3, pp. 523-532, 2001.

[12] C. X. Wu and M. Ma, "Embedding problem of fuzzy number space. I," Fuzzy Sets and Systems, vol. 44, no. 1, pp. 33-38, 1991.

[13] G. A. Anastassiou, "Rate of convergence of fuzzy neural network operators, univariate case," Journal of Fuzzy Mathematics, vol. 10, no. 3, pp. 755-780, 2002.

[14] G. Anastassiou, Quantitative Approximations, Chapman \& Hall/CRC, Boca Raton, Fla, USA, 2001.
[15] G. A. Anastassiou, Fuzzy Mathematics: Approximation Theory, vol. 251 of Studies in Fuzziness and Soft Computing, Springer, Berlin, Germany, 2010. 


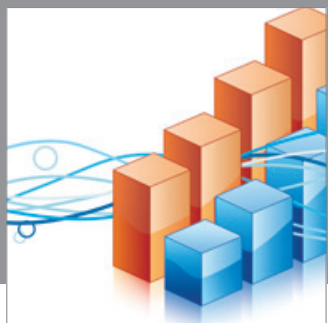

Advances in

Operations Research

mansans

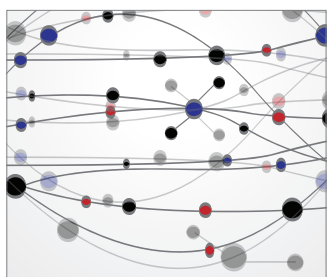

The Scientific World Journal
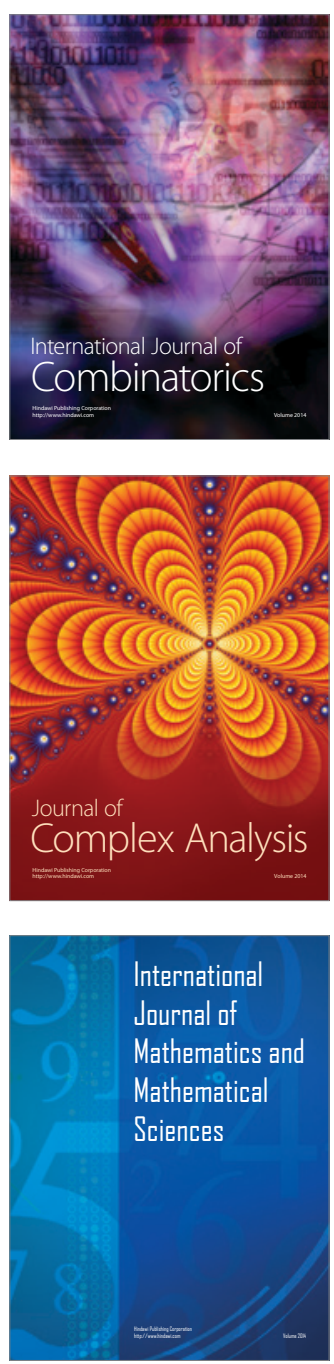
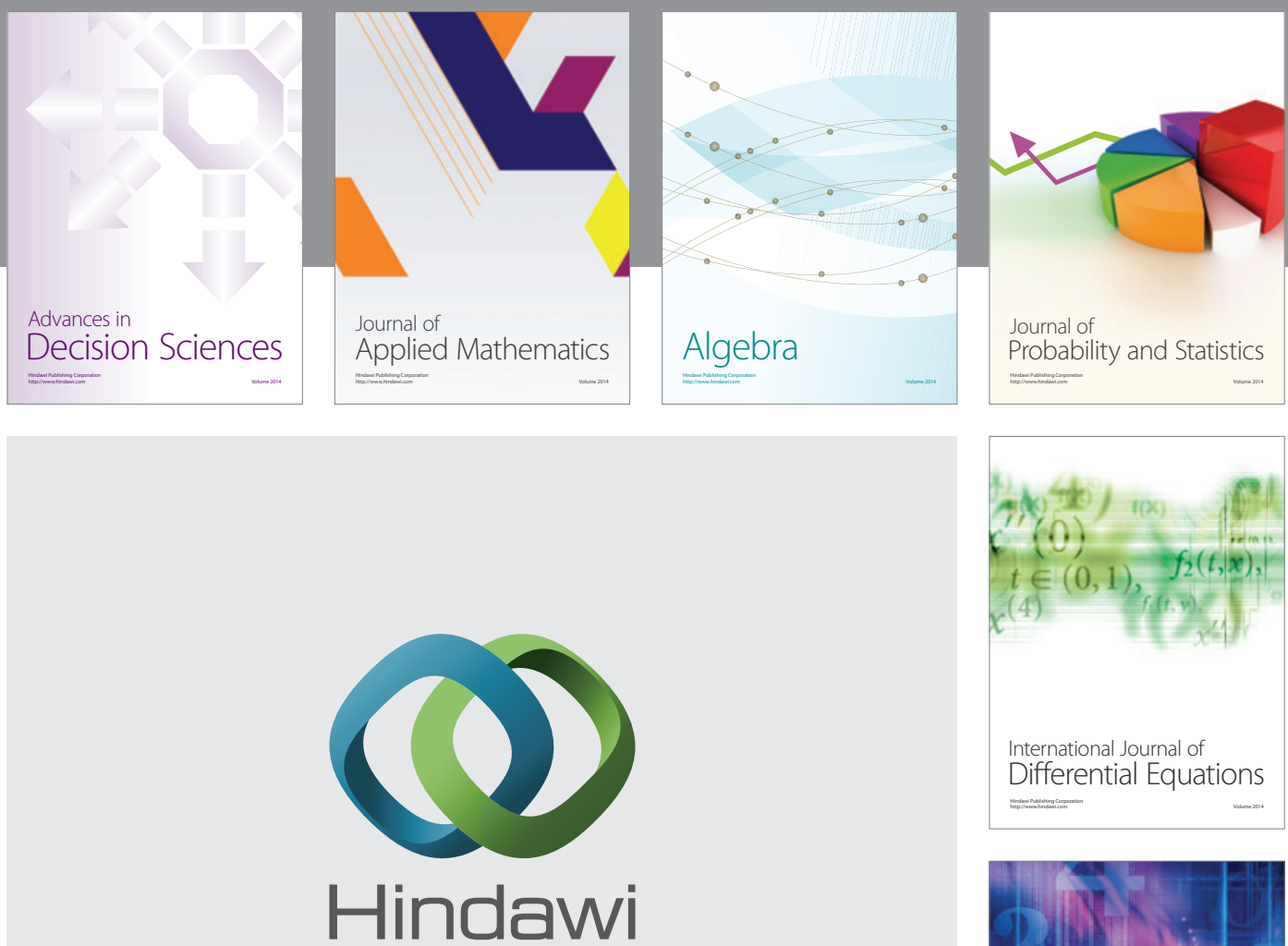

Submit your manuscripts at http://www.hindawi.com
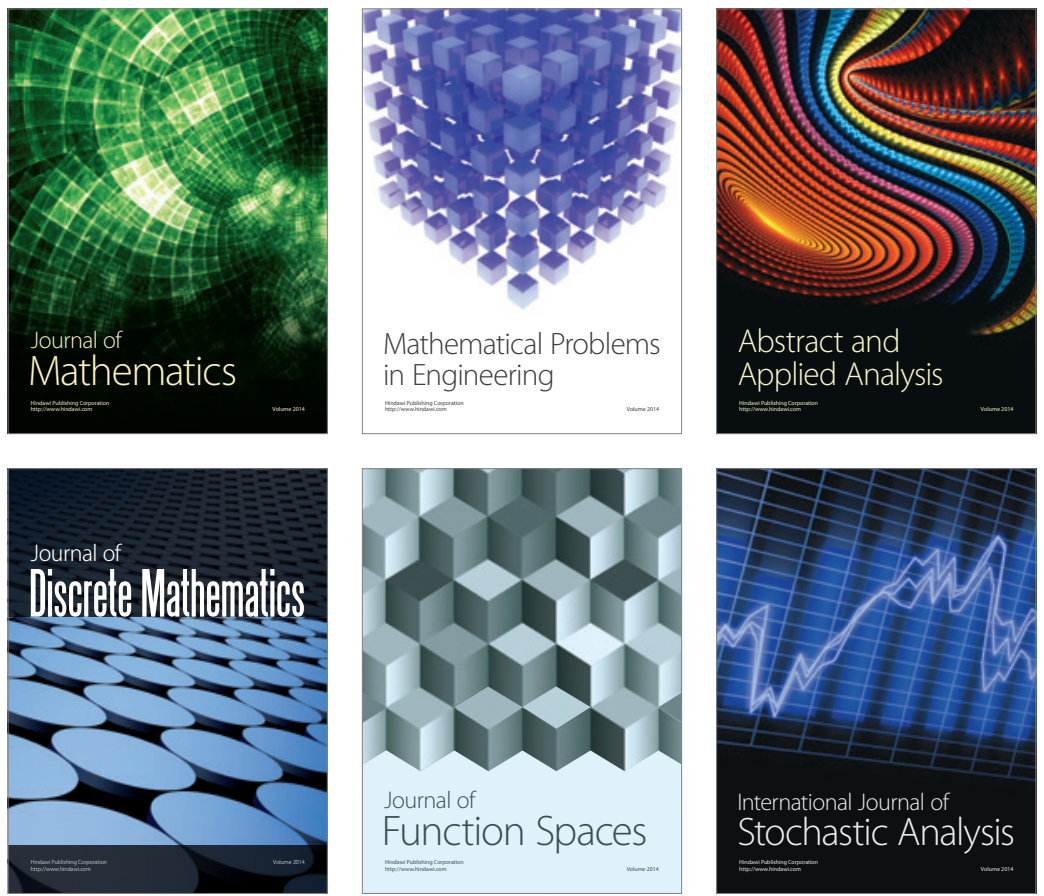

Journal of

Function Spaces

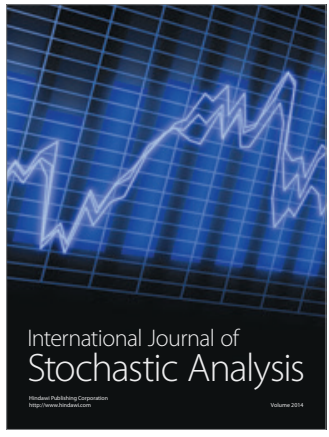

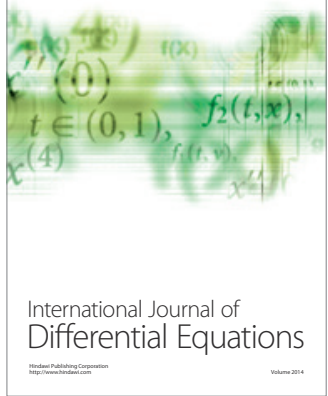
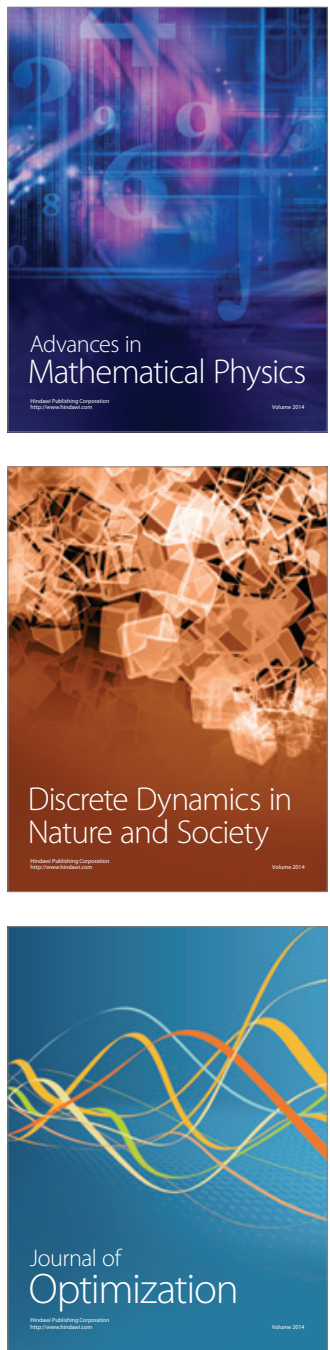